\title{
Superimposed High Frequency J et Ventilation Minimises Diaphragm, Bronchus, and Mediastinum Motion during One-Lung Flooding
}

\author{
Lesser $T^{1 *}$, Wolfram $\mathrm{F}^{1}$, Braun $\mathrm{C}^{2}$ and Gottschall \\ $\mathbf{R}^{3}$ \\ ${ }^{1}$ Department of Thoracic and Vascular Surgery, SRH \\ Wald-Klinikum Gera, Teaching Hospital of J ena \\ University Hospital, Strasse des Friedens 122, Gera \\ D-07548, Germany \\ ${ }^{2}$ Central Experimental Animal Facility, University \\ Hospital J ena, Location Dornburger Strasse 23a, J ena \\ D-07743, Germany \\ ${ }^{3}$ Doctor Emeritus, Department of Anaesthesiology and \\ Intensive Care, J ena University Hospital, Am Klinikum 1, \\ J ena D-07747, Germany \\ *Correspondling author: Lesser T, Department of \\ Thoracic and Vascular Surgery, SRH Wald-Klinikum \\ Gera, Teaching Hospital of J ena University Hospital, \\ Strasse des Friedens 122, Gera D-07548, Germany
}

Received: J uly 01, 2021; Accepted: J uly 26, 2021; Published: August 02, 2021

\begin{abstract}
Background: One-Lung Flooding (OLF) represents an ideal acoustic pathway for focused ultrasound ablation of lung tumours. Despite stabilization of the adjacent hemidiaphragm by OLF, standard Pressure-Controlled Ventilation (PCV) of the contralateral lung causes an unacceptable movement of the flooded lung. We examined whether Superimposed High Frequency Jet Ventilation (SHFJV) reduces lung motion compared to PCV during OLF.

Methods: The study included 15 pigs: 10 underwent OLF; 5 controls underwent two-lung ventilation without OLF. Using ultrasound, diaphragm displacement on the flooded lung side was measured during PCV and SHFJV in the left lateral (LLP), Supine (SP), and Right Lateral Positions (RLP). Bronchus and mediastinum displacements were measured in the right lateral position.

Results: Diaphragm displacement on the flooded lung side was significantly reduced during SHFJV, compared with PCV, in all animal positions (LLP: $7 \mathrm{~mm}$ [4.75-8.0] vs. $17 \mathrm{~mm}$ [14.75-19.0], $P=0.0039$; SP: $4 \mathrm{~mm}$ [3.75-4.25] vs. $17 \mathrm{~mm}$ [16.0-18.5], $\mathrm{P}=0.0039$; RLP: $8 \mathrm{~mm}$ [5.75-9.0] vs. $20 \mathrm{~mm}$ [14.0-23.25], $\mathrm{P}=0.0078$ ). Displacement of both the bronchus and mediastinum were significantly reduced during SHFJV, compared with PCV, in RLP (bronchus: 2.0mm [1.75-2.25] vs. 3.0mm [2.75-3.0], $\mathrm{P}=0.027$; mediastinum: 4.5mm [4.0-5.0] vs. $10 \mathrm{~mm}[7.0-10.0]$, $\mathrm{P}=0.0078$.
\end{abstract}

Conclusion: Thus, SHFJV minimises diaphragm, bronchus, and mediastinum motion during OLF, which is a prerequisite for effective lung tumour ablation.

Keywords: One-lung flooding; Superimposed high frequency jet ventilation; Lung motion; Focused ultrasound ablation; Animal model

\section{Introduction}

One-Lung Flooding (OLF) was developed to enable complete lung sonography of the flooded lung $[1,2]$. This novel method provides new diagnostic and therapeutic options for clarifying small pulmonary nodules and performing focused ultrasound ablation of lung tumours $[3,4]$. For effective tumour ablation, especially when using focused ultrasound, movement of the target lesion should be minimized [5]. One drawback of OLF is the requirement for one-lung ventilation of the contralateral lung, which usually involves standard Pressure-Controlled Ventilation (PCV). Disadvantages of PCV include diaphragm movement on the ventilated side. During OLF, alveolar gas in one lung is completely replaced with saline solution. Consequently, OLF simultaneously fulfils two essential requirements for lung tumour ablation. First, the flooded lung represents an ideal acoustic pathway for insonation of ultrasound using a transcutaneous approach. Second, the flooded lung is incompressible, thereby stabilizing the adjacent hemidiaphragm. Previously, we showed that diaphragm motion was significantly reduced on the flooded side during OLF, compared with standard two-lung ventilation [6]. However, immobilization of the lung by OLF, especially in the vicinity of the mediastinum, is not yet satisfactory. We detected residual hemidiaphragm motion with a maximum displacement of $15 \mathrm{~mm}$ close to the ventilated lung during OLF. The PCV of the contralateral lung is responsible for this because PCV causes both the diaphragm movement on the ventilated side that is transmitted to the opposite side and the mediastinal displacement toward the flooded lung.

By contrast, High Frequency Jet Ventilation (HFJV) utilizes low tidal volumes $(1-3 \mathrm{~mL} / \mathrm{kg})$ at high frequencies $(>100$ cycles/ $\mathrm{min}$ ), which reduce thoracoabdominal motion $[7,8]$. The technique has been used during thoracic surgery, extracorporeal shockwave lithotripsy, cardiac ablation, and percutaneous tumour ablation procedures to reduce target-lesion motion and optimize therapy [911]. The combination of OLF and contralateral lung Superimposed High Frequency Jet Ventilation (SHFJV) may prevent even small movements of the diaphragm, mediastinum, and lung.

In this study, we used a pig model to examine whether SHFJV of the non-flooded lung reduces diaphragm, bronchus, and mediastinum motion of the flooded side, compared with PCV.

\section{Materials and Methods}

After the protocol was approved by the Veterinary Department of the Thuringian State Authority for Food Protection and Fair Trading 
(TLLV Reg. 22-2684-04-WKG-16-002), this study was performed in laboratories at the Central Experimental Animal Facility, University Hospital Jena. The animals were maintained in groups and housed at the facility for 4 days before the study to acclimate them to the surroundings. All procedures were performed in compliance with the National Animal Protection Act.

\section{Animal preparation}

Fifteen juvenile female pigs (German Landrace), with a mean age of 12.4 weeks (range: 11-14 weeks) and mean weight of $37 \mathrm{~kg}$ (range: $35-40 \mathrm{~kg}$ ), were included in this study. Before the experiments began, each animal was confirmed to be in good health by a veterinarian.

Ketamine $(25 \mathrm{mg} / \mathrm{kg})$ and midazolam $(0.2 \mathrm{mg} / \mathrm{kg})$ were administered intramuscularly as premedicants to each pig. General anaesthesia was induced by injecting propofol $(3 \mathrm{mg} / \mathrm{kg})$ and fentanyl $(2.7 \mu \mathrm{g} / \mathrm{kg}) v i a$ a peripheral vein and maintained with a continuous intravenous infusion of propofol $(6 \mathrm{mg} / \mathrm{kg} / \mathrm{h})$ and hourly boluses of fentanyl $(2.7 \mu \mathrm{g} / \mathrm{kg})$. After onset of anaesthesia, the pigs were intubated transorally using a single-lumen endotracheal tube (6.5ID Dahlhausen, Köln, Germany). Pancuronium bromide (0.06mg/ $\mathrm{kg}$ ) was administered intravenously every hour as a neuromuscular blocking agent, and mechanical ventilation was performed using an intensive care unit ventilator (Servo 900C, Siemens AG, Erlangen, Germany) with these pressure-controlled settings: fraction of inspired oxygen $\left(\mathrm{FiO}_{2}\right)$ : 0.4 ; Pinsp $15 \mathrm{~cm} \mathrm{H}_{2} \mathrm{O}$; inspiratory to expiratory (I:E) ratio: $1: 1.9$; respiratory rate: 20 breaths $/ \mathrm{min}$; and positive endexpiratory pressure: $4 \mathrm{~cm} \mathrm{H}_{2} \mathrm{O}$. These settings were maintained throughout the experiment (and adjusted as necessary to maintain an end-tidal $\mathrm{CO}_{2}$ of $35-45 \mathrm{mmHg}$ ), except for the $\mathrm{FiO}_{2}$, which was increased to 1.0 for denitrogenation of the lungs before OLF.

An arterial catheter (Arterial Leader Cath, 2.7 Fr; Vygon, Ecouen, France) was placed using sterile technique and advanced $10 \mathrm{~cm}$ into the central common carotid artery for haemodynamic monitoring. With the pig in the supine position (SP), a 35-Fr left-sided Double Lumen Endobronchial Tube (DLT) designed for use in pigs and specifically made for this study (Medicoplast International $\mathrm{GmbH}$, Illingen, Germany) was placed using an airway exchange catheter (11.0 Fr, $100 \mathrm{~cm}$, extra-firm with a soft tip; COOK Deutschland $\mathrm{GmbH}$, Mönchengladbach, Germany). Correct position of the DLT was confirmed by fibreoptic bronchoscopy (BF 3C30; Olympus, Tokyo, Japan). A cuff controller (VBM Medizintechnik GmbH, Sulz a.N., Germany) was used to maintain a constant pressure of $50 \mathrm{~cm}$ $\mathrm{H}_{2} \mathrm{O}$ within both the endobronchial and tracheal cuffs.

All animals were connected to a Datex monitor (Datex AS3 Monitoring System; Datex-Ohmeda Corp., Helsinki, Finland) for continuous assessment of vital signs, including haemodynamic and respiratory parameters. The blood gas measurements were analysed using a blood gas analyser (Rapidpoint 405, Siemens Healthcare, Erlangen, Germany).

\section{Experimental protocol}

The experimental (OLF) and control (CO) groups were studied successively. The OLF group animals initially underwent two-lung ventilation with a $\mathrm{FiO}_{2}$ of 1.0 for 20 minutes to denitrogenate the lungs; this was necessary to achieve complete OLF. The animals were then placed in the left lateral decubitus position, with the lung to be flooded in the dependent position, and the left (bronchial) lumen of the DLT was disconnected from the ventilator. The infusion system was immediately connected to the left limb of the DLT, and the left lung was slowly filled (single filling) with degassed, warmed $\left(37^{\circ} \mathrm{C}\right)$ isotonic saline flowing passively from an infusion bottle suspended $50 \mathrm{~cm}$ above heart level. The volume to be infused was estimated as one-half the functional residual capacity of the lungs $(12.5 \mathrm{~mL} / \mathrm{kg})$. Complete saline filling was monitored with transcutaneous lung ultrasound. An endobronchial catheter was used to control the filling pressure. The animals remained in the Left Lateral Position (LLP) for $30 \mathrm{~min}$, after which they were placed in the SP for $30 \mathrm{~min}$, followed by $30 \mathrm{~min}$ in the Right Lateral Position (RLP). While the animals were in each position, the right lung was ventilated successively with PCV and SHFJV, each for 15 minutes. The order of each type of ventilation was determined by randomization before the experiment. Displacement of the left hemidiaphragm was determined by sonographic imaging during both types of ventilation in each body position. In addition, displacement of both the mediastinum and the left bronchus were sonographically imaged while animals were placed in RLP.

During PCV, the ventilator settings were maintained as described above, whereas during SHFJV the jet ventilator (TwinStream ${ }^{\mathrm{TM}}$, Carl Reiner GmbH, Vienna, Austria; Figure 1) was connected to the right (tracheal) lumen of the DLT using a special adapter (jet converter, I.D. 15mm; Carl Reiner GmbH, Vienna, Austria; Figure 2). Based on the results of a pilot study to determine the optimal jet ventilator settings, low and high frequencies were set at $20 \mathrm{~min}^{-1}$ and $200 \mathrm{~min}^{-1}$, with basic/outlet driving pressures of 0.9 and 0.4 bar and I:E ratios of $1: 2$ and $1: 1 . \mathrm{FiO}_{2}$ was set at 0.4 . The driving pressure for the low frequency jet stream was increased by 0.1-0.2 bar for a short time when the end-tidal $\mathrm{CO}_{2}$ exceeded $45 \mathrm{mmHg}$. Bias flow of warmed, humidified gas $\left(\mathrm{FiO}_{2} 0.4\right)$ at $20 \mathrm{lpm}$ was connected to the jet converter.

Blood gas analyses were performed during both types of ventilation in each body position.

In the CO group, two-lung ventilation (TLV) using PCV via a DLT was performed for 90 minutes in all animals. They did not undergo OLF. Every 30 minutes, the animal's position was changed (LLP, SP, and RLP), and measurements of diaphragm motion were performed from sonographic images as in the OLF group.

\section{Sonography}

Sonographic examinations were performed using a sonographic system (Flex Focus 800; BK Medical, Arhus, Denmark) with curved arrays (diaphragm: 6-2 MHz, 8820e; bronchus and mediastinum: 10-4 $\mathrm{MHz}, 8815)$. Left hemidiaphragm displacement was measured via an anterior subcostal approach. The ultrasound transducer was placed in the anterior axillary line and directed medially, cranially, and dorsally, so the ultrasonic beam reached the dome of the diaphragm. Images were optimised for frame rate using a single focus position, reducing depth and sector width. An M-mode cursor was placed to obtain the best possible alignment with the axis of actual diaphragm displacement. The M-mode view was stored on video, which was frozen if at least 2 respiratory cycles were recorded. Differences between end-expiratory and end-inspiratory levels were measured on the M-mode images. These measurements were repeated three times, all of which were obtained by a single operator with extensive experience in lung ultrasound. To reduce measurement errors, the 
ventilation methods were switched (from PCV to SHFJV or vice versa) while the transducer was held in the same position. These measurements were performed with the animals in all three positions (LLP, SP, and RLP).

Bronchus and mediastinum displacements were measured via an intercostal approach with the animals in RLP. An ultrasound transducer was placed in the midaxillary line craniocaudally (crossing the ribs), so the ultrasound beam provided a longitudinal view of the left lower lobe bronchus, and the contralateral pleura was visible. The M-mode cursor was placed to obtain the best possible alignment with the bronchus. Bronchus and mediastinum displacements were measured as changes in the coronal plane on M-mode imaging. The transducer was held in the same position while the ventilation methods were switched.

\section{Statistical methods}

All data were analysed using MedCalc Statistical Software, version 19.1.7 (MedCalc Software bv, Ostend, Belgium), and nonnormal distributions were confirmed using a Q-Q plot. Therefore, the data are presented as median and interquartile range. Mann-Whitney $\mathrm{U}$ test was used to analyses differences between independent groups (i.e., OLF and CO groups). Differences between ventilation methods (i.e., PCV vs. SHFJV) and between animal positions during each ventilation method were analysed using Wilcoxon test. Statistical significance (two sided) was set at a P value less than 0.05 .

\section{Results}

In total, 14 animals were examined in this study: 5 served as controls and 9 were included in the OLF group. One animal died of acute cardiac death prior to OLF. No animals died during OLF.

Diaphragm displacement on the flooded lung side was significantly reduced during SHFJV, compared with $\mathrm{PCV}$, in all animal positions (LLP: $7 \mathrm{~mm}$ [4.75-8.0] vs. $17 \mathrm{~mm}$ [14.75-19.0], $\mathrm{P}=0.0039$; SP: $4 \mathrm{~mm}$ [3.75-4.25] vs. $17 \mathrm{~mm}$ [16.0-18.5], $\mathrm{P}=0.0039$; RLP: $8 \mathrm{~mm}$ [5.75-9.0] vs. $20 \mathrm{~mm}$ [14.0-23.25], $\mathrm{P}=0.0078)$. During SHFJV, the amount of diaphragm displacement was least in SP (vs. LLP: $\mathrm{P}=0.015$; vs. RLP:

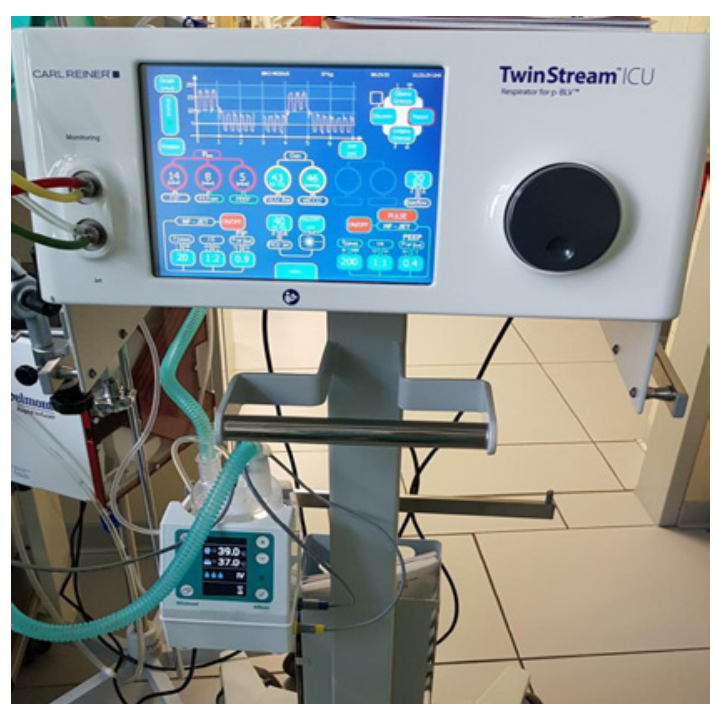

Figure 1: The jet ventilator TwinStream ${ }^{\mathrm{TM}}$ (Carl Reiner GmbH, Vienna, Austria). The ventilation mode "bronchoscopy" was used for SHFJV.

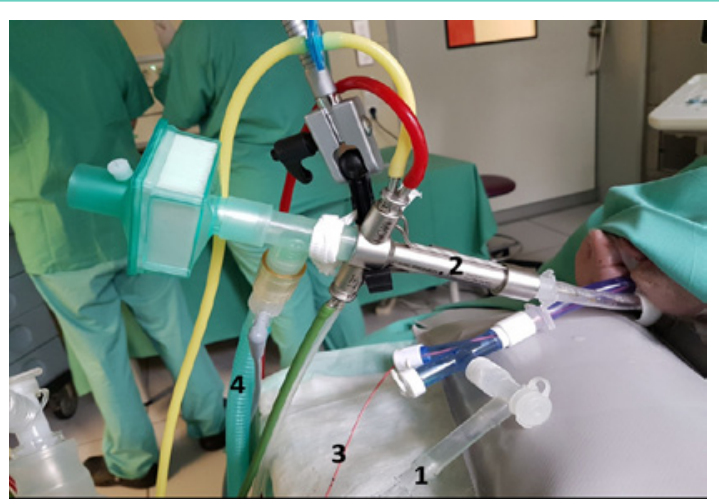

Figure 2: SHFJV of the right lung using the jet converter connected to the right (tracheal) limb of the double lumen endobronchial tube. The left lung was filled with saline via a special adaptor. 1) Infusion system connected via a special adaptor to the left limb for one-lung flooding with saline; 2) Jet converter for Superimposed High-Frequency Jet Ventilation connected to the right limb of the double-lumen tube; 3) Endobronchial catheter; 4) Bias flow of gas supplied via a t-piece connected to the jet converter.

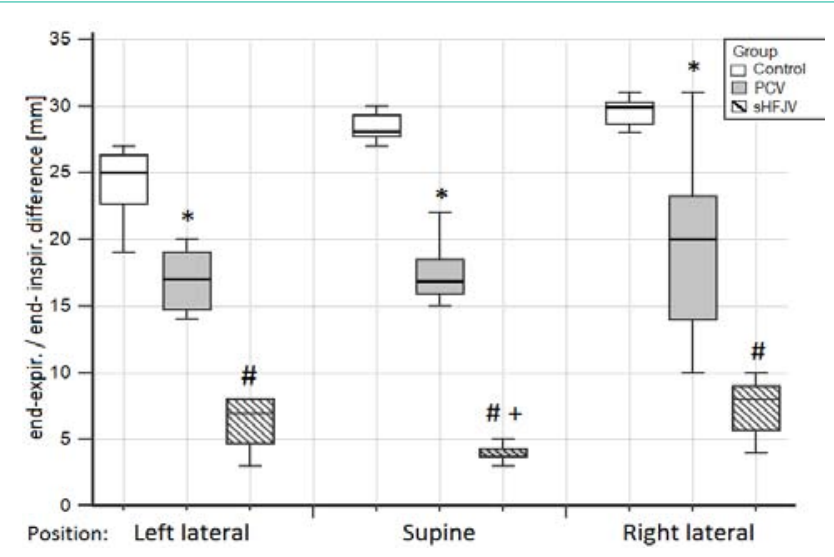

Figure 3: Diaphragm displacement of the left hemidiaphragm (flooded lung side) after one-lung flooding during Pressure-Controlled Ventilation and Superimposed High Frequency Jet Ventilation in different animal positions. White box, control group with Pressure-Controlled Two-Lung Ventilation (Control); grey box, Pressure-Controlled Ventilation (PCV); box with slashes, Superimposed High Frequency Jet Ventilation (SHFJV); ${ }^{*} \mathrm{P}<0.05$ vs. corresponding CO; ${ }^{P} \mathrm{P}<0.05$ vs. corresponding $\mathrm{PCV}$; ${ }^{+} \mathrm{P}<0.05$ vs. SHFJV in left and right lateral positions.

$\mathrm{P}=0.0039$ ). Diaphragm displacement on the flooded lung side was significantly reduced during OLF and PCV, compared with TLV (CO group), in all animal positions (LLP: $17 \mathrm{~mm}[14.75-19.0]$ vs. $25 \mathrm{~mm}$ [22.75-26.25], $\mathrm{P}=0.0059$; SP: $17 \mathrm{~mm}[16.0-18.5]$ vs. $28 \mathrm{~mm}$ [27.7529.25], $\mathrm{P}=0.0025$; RLP: $20 \mathrm{~mm}$ [14.0-23.25] vs. $30 \mathrm{~mm}$ [28.75-30.25], $\mathrm{P}=0.016$, Figure 3 and 4).

Displacement of both the bronchus and mediastinum were significantly reduced during SHFJV, compared with PCV, in RLP (bronchus: $2.0 \mathrm{~mm}$ [1.75-2.25] vs. $3.0 \mathrm{~mm}$ [2.75-3.0], $\mathrm{P}=0.027$; mediastinum: $4.5 \mathrm{~mm}$ [4.0-5.0] vs. $10 \mathrm{~mm}$ [7.0-10.0], $\mathrm{P}=0.0078$; Figure 5 and 6).

\section{Discussion}

In this study, the effects of SHFJV on displacement of the diaphragm, bronchus, and mediastinum on the flooded lung side 

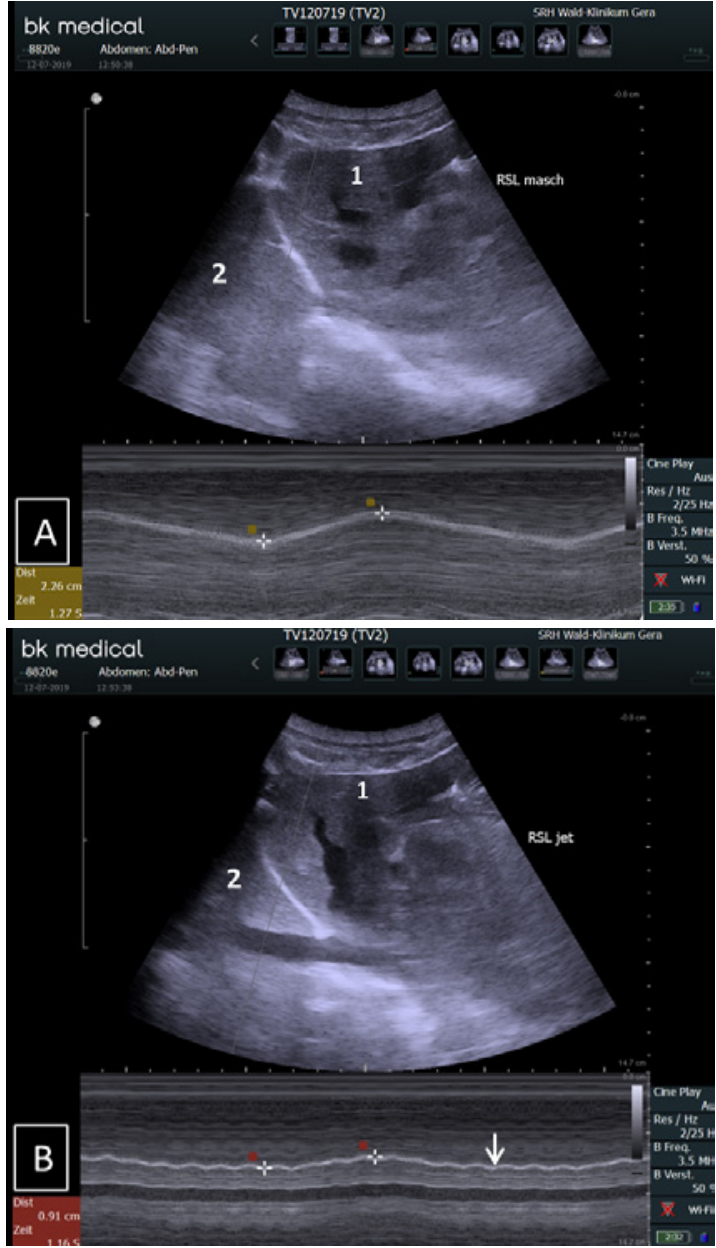

Figure 4: Diaphragm M-mode ultrasound images from the anterior subcostal view for measuring left hemidiaphragm (flooded lung side) motion during Pressure-Controlled Ventilation (A) and Superimposed High Frequency Jet Ventilation $(B)$ in the right lateral position. The transducer was held in the same position while ventilation methods were changed. The motion curve is jagged during jet ventilation because of the high-frequency jet stream (arrow) 1) Liver; 2) Flooded lung.

during OLF were compared with the effects of PCV. The key result was that SHFJV significantly reduced motion of all three structures.

OLF itself significantly reduces hemidiaphragm displacement on the flooded lung side during one-lung PCV. The saline-filled lung represents an incompressible mass that impedes movement of the ipsilateral hemidiaphragm. This study confirmed the results of our previous study, in which we found $78 \%$ reduction of diaphragm motion during OLF, compared with TLV. However, at that time we detected residual hemidiaphragm motion with a maximum displacement of $15 \mathrm{~mm}$ close to the ventilated lung during OLF [6]. This amount of displacement could be too high for safe and effective focused ultrasound ablation of tumours lying near the caudal mediastinum. The current study was therefore initiated to examine whether SHFJV could further reduce diaphragm motion on the flooded lung side.

The absolute end-expiratory and end-inspiratory motion values in the previous study [6] were lower than those in the current study

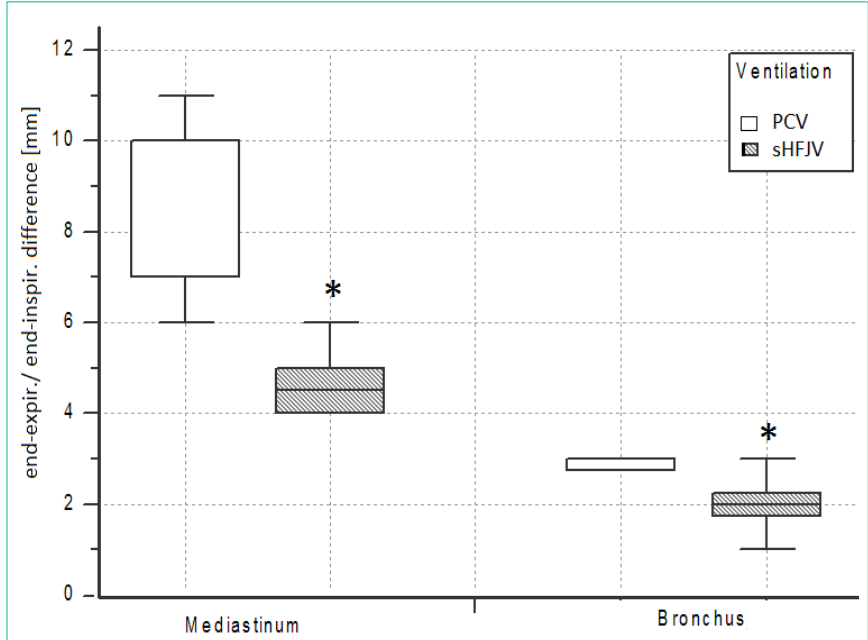

Figure 5: Bronchus and mediastinum displacements on the flooded lung side during Pressure-Controlled Ventilation and Superimposed High Frequency Jet Ventilation in the right lateral position. White box, Pressure-Controlled Ventilation (PCV); box with slashes, Superimposed High Frequency Jet Ventilation (SHFJV). *P $<0.05$ vs. Pressure-Controlled Ventilation. Error bars for SHFJV mediastinum and for PCV bronchus overlap with box limits.

because the right lung was flooded with a higher filling volume $(15.5 \mathrm{~mL} / \mathrm{kg})$, and the animals were ventilated with a volumecontrolled setting using a lower tidal volume $(10 \mathrm{~mL} / \mathrm{kg})$. In the present study, the left lung was flooded with $12.5 \mathrm{~mL} / \mathrm{kg}$ saline, and the contralateral lung was ventilated with PCV and a target tital volume of $15 \mathrm{~mL} / \mathrm{kg}$. Furthermore, during right lung flooding, the accessory lobe in the mediastinal recessus is filled, which arises from the right bronchus and lies directly on the diaphragm. Therefore, the area of the diaphragm covered by flooded lung is larger with right lung flooding than with left lung flooding. Additionally, motion of the right diaphragm is restricted by the underlying liver.

The passive breath hold method was used to overcome the motion problem during liver tumour ablation $[12,13]$. Passive breath hold is however associated with a long treatment time and decreased efficiency, as the sonication is performed non-continuously.

HFJV has proven effective for reducing thoracoabdominal motion, but randomised controlled studies are needed to prove its superiority to standard ventilation [10]. There are many clinical settings, such as extracorporeal shockwave lithotripsy, cardiac ablation, and computed tomography-guided lung tumour ablation with radiofrequency, in which HFJV has been used to reduce target-lesion motion [14-16]. To prevent the possibility of $\mathrm{CO}_{2}$ retention under high-frequency jet ventilation [17] we used a jet ventilator that allows simultaneous application of two different jet streams (low frequency and high frequency), resulting in pulsatile bi-level ventilation. The low frequency is primarily responsible for $\mathrm{CO}_{2}$ elimination, while the high frequency is responsible for oxygenation. With SHFJV, superimposition of the low-frequency jet induces additional gas flow, resulting in thoracic inspiratory and expiratory movements similar to those during conventional ventilation. The low-frequency jet stream is responsible for lung and diaphragm movements and could be a disadvantage for immobilization of the flooded contralateral lung. Nevertheless, our results showed that SHFJV led to marked reduction 

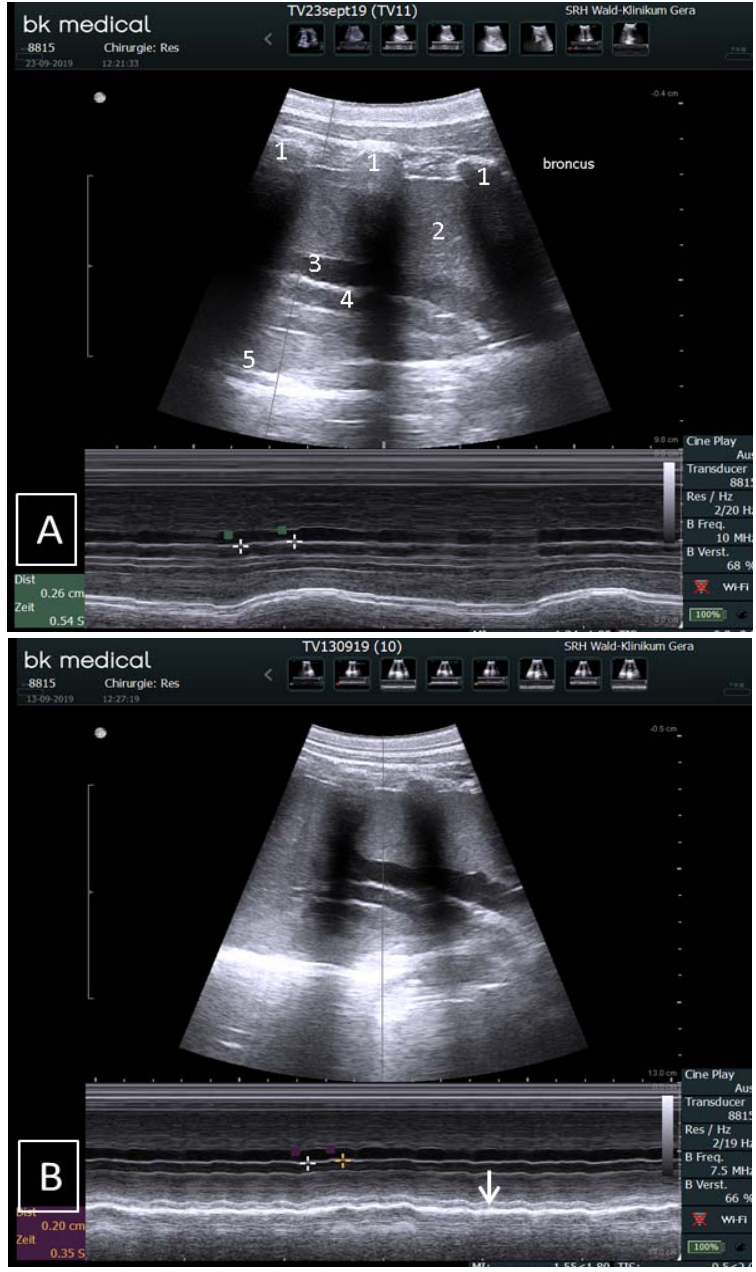

Figure 6: Lung M-mode ultrasound imaging from a lateral transcostal view for measuring displacement the bronchus and mediastinum on the flooded lung side during Pressure-Controlled Ventilation (A) and Superimposed High Frequency Jet Ventilation (B) in the right lateral position. Distance markers indicate bronchus displacement. Note the reduced mediastinum displacement during Superimposed High Frequency Jet Ventilation, compared with pressure-controlled ventilation (arrow). 1) Ribs; 2) Flooded left lung; 3) Pulmonary artery; 4) Left lower bronchus; 5) Pleura reflex of the opposite lung.

of diaphragm, bronchus, and mediastinum displacement when using a basic/outlet driving pressure of 0.9 bar for the low-frequency jet stream. The benefits of jet ventilation regarding motion reduction are negated if higher pressures are used. The empirically found jet parameters in the pilot study represent a compromise between tolerable gas exchange and effective movement reduction. In the future, we plan to examine the effects of SHFJV on haemodynamics and gas exchange during OLF in different animal positions.

Our results showed that SHFJV significantly reduced diaphragm, bronchus, and mediastinum displacement in all body positions. This is important because the required position for interventional targeting is based on available instrumentations and anatomic locations. Depending on both the tumour location and the method of ablation (magnetic resonance-guided or ultrasound-guided high-intensity focused ultrasound ablation) the flooded lung can be dependent or non-dependent.
The ability of SHFJV to reduce mediastinum and central bronchus movements is also an important result. Central tumours are usually located close to important functional structures, such as the heart, pulmonary vessels, and bronchi. Imprecise ablation of central tumours because of uncontrolled motion can cause life-threatening damage to these structures $[18,19]$.

This study had some limitations that should be addressed. For example, motion of the mediastinum and bronchus cannot be measured on the ventilated lung side by ultrasound, which prevented direct comparisons of displacement between the flooded and ventilated sides. Furthermore, we assume that the observed decreases in diaphragm, mediastinum, and bronchus movements are transferable to tumours. Ideally, tumour movement would be assessed, but an appropriate pig tumour model is not available. Additionally, although we attempted to reduce measurement variability between the two ventilation methods by using a fixed transducer position while switching between SHFJV and PCV within the same animal, the transducer position may have differed slightly between animals.

\section{Conclusions}

In summary, we examined whether SHFJV during OLF reduced diaphragm, bronchus, and mediastinum motion on the flooded lung side, compared with PCV. Our results clearly demonstrated that SHFJV minimized the motion of these structures. The combination of OLF in tumour-containing lung and SHFJV in the contralateral lung appears to provide optimal conditions for effective lung tumour ablation.

\section{Declarations}

Ethics approval and consent to participate.

The study was approved by the Veterinary Department of the Thuringian State Authority for Food Protection and Fair Trading (TLLV Reg. 22-2684-04-WKG-16-002).

Consent for publication: All authors have given their consent for the manuscript to be published.

Availability of data and materials: All of the data and materials are available.

Author contributions: Conceptualization: Thomas Lesser, Reiner Gottschall; Formal analysis: Thomas Lesser, Frank Wolfram; Funding acquisition: Thomas Lesser, Frank Wolfram; Investigation: Thomas Lesser, Reiner Gottschall, Conny Braun; Project administration: Thomas Lesser; Writing - original draft: Thomas Lesser; Writing review and editing: Reiner Gottschall, Frank Wolfram.

Acknowledgments: The authors gratefully acknowledge technical support provided by Mr. R. Kölbl and Mr. M Frank (Carl Reiner GmbH, Vienna, Austria). We also want to acknowledge Dipl. Ing. F. Sick, Medicoplast International GmbH, Illingen, Germany, for providing the double-lumen endobronchial tubes. We likewise thank the team at the Central Experimental Animal Facility, University Hospital Jena, especially Mrs. Dobermann, Mrs. Goebel, and Mr. Wuckelt, for their assistance with this project. We also thank PD Dr. U. Leder, MBA, A. Peuke, and M. Lechner from SRH Wald-Klinikum Gera for their support. 


\section{References}

1. Lesser $T$, Klinzing $S$, Schubert $H$, Klein U, Bartel M. Lung flooding-a new method for complete lung sonography. Res Exp Med. 1998; 198: 83-91.

2. Lesser TG, Schubert $H$, Bischoff $S$, Wolfram F. Lung flooding enables efficien lung sonography and tumour imaging in human ex vivo and porcine in vivo lung cancer model. Eur J Med Res. 2013; 18: 23.

3. Wolfram F, Reichenbach JR, Lesser T. An ex vivo human lung model for ultrasound-guided high-intensity focused ultrasound therapy using lung flooding. Ultrasound Med Biol. 2014; 40: 496-503.

4. Lesser T, Petersen I, Pölzing F, Wolfram F. One-Lung Flooding Enables Ultrasound-Guided Transthoracic Needle Biopsy of Pulmonary Nodules with High Sensitivity. Ultrasound Med Biol. 2018; 44: 1556-1562.

5. Schwaab J, Kurz C, Sarti C, Bongers A, Schoenahl F, Bert C, et al. First Steps toward Ultrasound-Based Motion Compensation for Imaging and Therapy: Calibration with an Optical System and 4D PET Imaging. Front Oncol. 2015; 5: 258.

6. Lesser T, Schubert H, Güllmar D, Reichenbach JR, Wolfram F. One-lung flooding reduces the ipsilateral diaphragm motion during mechanical ventilation. Eur J Med Res. 2016; 21: 9.

7. Evans E, Biro P, Bedforth N. Jet ventilation. Contin Educ Anaesth Crit Care Pain. 2007; $7: 2-5$.

8. Galmén K, Harbut P, Freedman J, Jakobsson JG. The use of high-frequency ventilation during general anaesthesia: an update. F1000Res. 2017; 6: 756.

9. Mucksavage P, Mayer WA, Mandel JE, Van Arsdalen KN. High-frequency jet ventilation is beneficial during shock wave lithotripsy utilizing a newer unit with a narrower focal zone. Can Urol Assoc J. 2010; 4: 333-335.

10. Galmén K, Harbut P, Freedman J, Jakobsson JG. High frequency je ventilation for motion management during ablation procedures, a narrative review. Acta Anaesthesiol Scand. 2017; 61: 1066-1074.

11. Buchan T, Walkden M, Jenkins K, Sultan P, Bandula S. High-frequency je ventilation during cryoablation of small renal tumours. Cardiovasc Intervent Radiol. 2018; 41: 1067-1073.
12. Wu F, Wang ZB, Chen WZ, Zou JZ, Bai J, Zhu H, et al. Advanced hepatocellular carcinoma: Treatment with high-intensity focused ultrasound ablation combined with transcatheter arterial embolization. Radiology. 2005; 235: 659-667.

13. Kopelman D, Inbar Y, Hanannel A, Dank G, Freundlich D, Perel A, et al. Magnetic resonance-guided focused ultrasound surgery (MRgFUS). Four ablation treatments of a single canine hepatocellular adenoma. HPB (Oxford). 2006; 8: 292-298.

14. Abderhalden S, Biro P, Hechelhammer L, Pfiffner R, Pfammatter T. CTguided navigation of percutaneous hepatic and renal radiofrequency ablation under high frequency jet ventilation: feasibility study. J Vasc Interv Radiol. 2011; 22: 1275-1278.

15. Denys A, Lachenal Y, Duran R, Chollet-Rivier M, Bize P. Use of highfrequency jet ventilation for percutaneous tumor ablation. Cardiovasc Intervent Radiol. 2014; 37: 140-146.

16. Chung DYF, Tse DML, Boardman P, Gleeson FV, Little MW, Scott SH, et al. High-frequency jet ventilation under general anesthesia facilitates CTguided lung tumor thermal ablation compared with normal respiration under conscious analgesic sedation. J Vasc Interv Radiol. 2014; 25: 1463-1469.

17. Galmén K, Jakobsson JG, Freedman J, Harbut P. High Frequency Jet Ventilation during stereotactic ablation of liver tumours: an observational study on blood gas analysis as a measure of lung function during general anaesthesia. F1000Res. 2019; 8: 386.

18. Heerink WJ, de Bock GH, de Jonge GJ, Groen HJ, Vliegenthart R, Oudkerk $\mathrm{M}$. Complication rates of CT-guided transthoracic lung biopsy: meta-analysis. Eur Radiol. 2017; 27: 138-148.

19. Liu Y, Zhang WW, He M, Gong C, Xie B, Wen X, et al. Adverse effect analysis of high-intensity focused ultrasound in the treatment of benign uterine diseases. Int J Hyperthermia. 2018; 35: 56-61. 